\title{
BMJ Open Ten-year time trends in preterm birth during a sociodemographic transition period: a retrospective cohort study in Shenzhen, China
}

\author{
Rui Ma (D) , ${ }^{1}$ Yali Luo, ${ }^{2}$ Jun Wang, ${ }^{1}$ Yanxia Zhou, ${ }^{1}$ Haiyang Sun, ${ }^{1}$ Xi Ren, ${ }^{1}$ Quan Xu, ${ }^{2}$ \\ Lian Zhang, ${ }^{3}$ Lingyun Zou (D) ${ }^{1}$
}

To cite: Ma R, Luo Y,

Wang J, et al. Ten-year time trends in preterm birth during a sociodemographic transition period: a

retrospective cohort study in Shenzhen, China. BMJ Open 2020;10:e037266. doi:10.1136/ bmjopen-2020-037266

- Prepublication history and additional materials for this paper is available online. To view these files, please visit the journal online (http://dx.doi. org/10.1136/bmjopen-2020037266).

Received 26 January 2020 Revised 07 September 2020 Accepted 08 September 2020

\section{Check for updates}

(c) Author(s) (or their employer(s)) 2020. Re-use permitted under CC BY-NC. No commercial re-use. See rights and permissions. Published by BMJ.

${ }^{1}$ Center for Big Data Research in Health, Shenzhen Baoan Women's and Children's Hospital, Jinan University, Shenzhen, China

${ }^{2}$ Department of Prevention \& Health Care, Shenzhen Baoan Women's and Children's Hospital, Jinan University, Shenzhen, China ${ }^{3}$ Department of Neonatology, Shenzhen Baoan Women's and Children's Hospital, Jinan University, Shenzhen, China

\section{Correspondence to}

Dr Lingyun Zou; lingyun.zou@gmail.com and Dr Lian Zhang;

zhliande@qq.com

\section{ABSTRACT}

Objectives To investigate time trends of preterm birth and estimate the contributions of risk factors to the changes in preterm birth rates over a decade (2009-2018) of transitional period in Shenzhen, China.

Design Retrospective cohort study between 2009 and 2018.

Setting All births in Baoan during January 2009 and December 2018 registered in the Shenzhen Birth Registry Database.

Participants 478044 live births were included with sociodemographic and medical records for both women and infants.

Outcome measures The incidence rate of preterm birth stratified by different maternal and infant characteristics. Multiple logistic regression was used to identify significant risk factors associated with preterm birth. The population attributable risk fraction of each factor was calculated to estimate its contribution to variations of preterm birth rate over the 10 years.

Results A total of 27829 preterm births from 478044 $(5.8 \%)$ live births were recorded and the preterm birth rate increased from $5.5 \%$ in 2009 to $6.2 \%$ in 2018 . Medically induced preterm birth rate increased from $2.0 \%$ in 2009 to $3.4 \%$ in 2018 while spontaneous preterm labour rate decreased from $3.3 \%$ to $2.7 \%$ over the decade years. Risk factors including multiple pregnancy $(0.28 \%$ increase) drove the rise of preterm birth rate, whereas changes in maternal educational attainment $(0.22 \%$ reduction) and prenatal care utilisation ( $0.45 \%$ reduction) had contributed to the decline in preterm birth rate.

Conclusions An uptrend of preterm birth rate was observed in an area under rapid sociodemographic transitions during 2009-2018 and the changes were associated with these sociodemographic transitions. Continued investments in girls' education and prenatal care have the potential of reducing preterm birth rate.

\section{INTRODUCTION}

Preterm birth, which is defined as birth of a neonate before 37 completed weeks of gestation, is a syndrome with many causes and multiple phenotypes. ${ }^{12}$ Globally, approximately 15 million neonates were born preterm in 2014, and over one million children die
Strengths and limitations of this study

- A complete and large-scale dataset of 480845 births across the recent 10-year period was analysed in this study to present long-term time trends in preterm birth and associated factors.

- Preterm births were classified into three specific subtypes including both spontaneous preterm births and iatrogenic preterm births to further examine the impact of risk factors on each type of preterm birth.

- Population attributable risk fraction was used to quantify the contributions of changes in risk factors to the variations of preterm birth rate before and after the two-child policy initiation.

- Preterm birth subtypes were unavailable in the Shenzhen Birth Registry Database and the classification was based on delivery mode and surgical indications.

- Possible risk factors including family income, maternal employment, maternal obesity and more were unavailable in the database and have not been analysed in the study.

each year due to preterm birth complications, contributing to approximately $16 \%$ of all deaths and $35 \%$ of newborn deaths in $2019 .{ }^{34}$ Despite decades of substantial research, the increasing prevalence of preterm birth continued to rise in many countries, resulting in a worldwide increase from $9.8 \%$ in the year of 2000 to $10.6 \%$ in the year of $2014 .^{5}$ Even fortunate survivors may experience lifetime disabilities, including neurodevelopmental and physical impairments, as well as behavioural effects, which impose a heavy burden on the family and society. ${ }^{6}$ The economic burden associated with preterm birth complications was at least US\$26.2 billion in the USA in 2005 and \$C587.1million in Canada in 2014. ${ }^{78}$ Addressing risk factors for preterm birth and determining the cause of the incremental incidence are critical to informing public health policies aimed at reducing the global burden 
of preterm births and achieving the Global Strategy for Women's, Children's and Adolescents' Health under the Sustainable Development Goals. ${ }^{9}$

Sociodemographic transitions have been shown to be associated with time trends in preterm birth, especially in areas during the industrialisation period. A cohort study from Bangladesh revealed that $27 \%$ of the decline in preterm birth rate could be attributed to the decrease in parity and expansion of maternal education during 1990-2014. ${ }^{10}$ Maternal age at delivery could explain the secular trends of preterm birth in Japan from 1979 to 2014 based on national birth data. ${ }^{11}$ During the Chilean sociodemographic transition period 1991-2012, the increase in advanced maternal age (35 years or older) was evaluated to significantly increase the risk of preterm birth. ${ }^{12}$ Economic inequalities were also found in relation to preterm births in four Brazilian birth cohort studies conducted between 1982 and 2011. ${ }^{13}$ A populationbased cohort study on births in Newcastle upon Tyne in Northern England over four decades confirmed the widened preterm birth gap between the most and least deprived socioeconomic groups. ${ }^{14}$

China accounted for $7.8 \%$ (1.17 million) of preterm births worldwide, with the second largest number of preterm neonates. ${ }^{5}$ From 2000 to 2014, the estimated preterm birth rate in China increased from $6.35 \%$ to $6.94 \% .^{5}$ As the first special economic zone located in the Pearl River Delta of South China, Shenzhen has undergone rapid urbanisation and has attracted millions of migrant labourers since the beginning of the 1980s. ${ }^{15}$ Baoan epitomises this urbanisation as the largest district in Shenzhen, with a more than 3.26 million year-end permanent population in 2018 and approximately 82\% are migrants from other parts of China. ${ }^{16}$ During 2012 and 2018, the de jure population of Baoan rapidly expanded from 2.68 million to 3.26 million, and the gross domestic product per capita grew from US $\$ 8556$ in 2008 to US $\$ 15981$ in $2017 .{ }^{16-18}$ In addition, the proportion of labourers in the tertiary sector of the economy increased from $15.7 \%$ in 2012 to $22.6 \%$ in $2017 .{ }^{16} 17$

However, limited studies on long-term time trends in preterm birth are available in areas under drastic sociodemographic transitions in China during recent decades. Transitions in the society and their contributions to the changes in preterm birth rate are still unclear. The present study was based on all births in Baoan during 2009-2018 registered in the Shenzhen Birth Registry Database. We assessed the temporal trends in preterm birth and associated risk factors among a large proportion of the migrant population. We further estimated the quantitative contributions of these factors to the changes in preterm birth rate over the last decade.

\section{METHODS}

\section{Study design and data collection}

This cohort study was based on data of all births in Baoan from 1 January 2009 to 31 December 2018, extracted from the Shenzhen Birth Registry Database, which has served as a system for birth registration and maternal and infant health management since 2000. ${ }^{19}$ Demographic and clinical records of both mothers and new-borns were available for the identification of preterm birth and risk factors. Only live births were included in this study and ineligible records were excluded to ensure the coherence and continuity of preterm birth rate calculations based on prior related research: (1) stillbirths or births with unknown results; (2) births with missing gestational age or gestational age $<22$ weeks or $>46$ weeks; and (3) births with missing maternal age or maternal age $<13$ years or $>50$ years. The flowchart of data selection is shown in the online supplemental figure $1 .{ }^{1520}$

\section{Patient and public involvement}

This study used routinely collected administrative health data and no patients were involved in the conception, design and conduct of the research. The results will be disseminated through open access publication.

\section{Ethics approval}

This study was approved by the Medical Ethics Committee of Shenzhen Baoan Women's and Children's Hospital, Jinan University. Data collection in the study were anonymous, and no individually identifiable information was available for the analysis.

\section{Definition and measurements}

Preterm birth is defined by the WHO as all births before 37 completed weeks of gestation or fewer than 259 days since the first day of a woman's last menstrual period. ${ }^{321}$ Based on gestational age, it was further classified as extremely preterm ( $<28$ weeks), very preterm (28-<32 weeks) and late preterm $(32-<37$ weeks). Preterm birth rate was calculated using the number of live births in a specific preterm category divided by all live births multiplied by 100 in a specific time period. ${ }^{21}$

Based on the delivery mode and surgical indications recorded in the database, we categorised preterm birth into three subtypes: two spontaneous preterm birth subtypes, including preterm premature rupture of membranes (PROM-PTB) and preterm labour (S-PTB), and the third subtype medically induced preterm birth (MI-PTB). PROM-PTB was defined as preterm birth with premature rupture of membranes, S-PTB was defined as non-PROM with vaginal deliveries and MI-PTB was defined as preterm birth with either induction of labour or caesarean section delivery but without PROM. ${ }^{20}$

Potential risk factors related to preterm birth were selected and analysed based on a literature review. ${ }^{10-14}$ Variables including gestational age, maternal age, maternal education, maternal ethnicity, immigrant, smoking, drinking, parity, delivery mode, fertility treatment, gestational age at the first prenatal care visit, number of prenatal care visits, gestational hypertension, gestational diabetes, pre-eclampsia or eclampsia, infant sex and date of delivery were included in our analysis. 
Numeric variables including gestational age, maternal age and gestational age at the first prenatal care visit were categorised into ordinal subgroups. We classified maternal education into three categories for a more balanced population and clear data interpretation: primary school and below, secondary and high school and college and above. ${ }^{22}$ Prenatal care utilisation is recommended by the Law of the People's Republic of China on Maternal and Infant Health Care with the initiation of antenatal care during the first trimester of pregnancy and consisting of five or more antenatal care visits. ${ }^{23}$ Three first visit trimester groups were generated based on the gestational age at the first prenatal care visit. ${ }^{24}$ The number of prenatal care visits was transformed into the prenatal care utilisation rate, by calculating the ratio between the actual number of visits and the recommended number of visits. The ratio was then classified into three groups: inadequate $(<50 \%)$, intermediate and appropriate $(50 \%-$ $110 \%)$ and adequate plus $(\geq 110 \%) .{ }^{24}$ To analyse the effect of the universal two-child policy, we classified births into two groups based on the time of delivery: births taking place before or within 9 months after the implementation of the universal two-child policy in October 2015 (June 2016) and births taking place 9 months after the policy. ${ }^{25}$

\section{Statistical analysis}

The $\chi^{2}$ test was used to evaluate significant differences in frequencies of both overall and subtypes of preterm birth between each maternal and infant group in this study. ${ }^{26}$ Annual overall preterm birth rates for each risk factor subcategory were calculated to present the temporal trends stratified by different characteristics over the decade. Yearly per cent compositions of each risk factor were calculated to show the changes in sociodemographic indicators. Sensitivity analysis was performed to examine changes in linear trends of annual overall preterm birth rates by calculating risk ratios, with the year 2009 as a reference. ${ }^{27}$ Multivariable binomial logistic regression models were applied to estimate adjusted odds ratios (AORs) and 95\% CIs of covariates for overall preterm birth and subtype-specific preterm birth. ${ }^{28}$ Possible independent variables were selected based on their significance in univariate analyses $(\mathrm{p}<0.05)$ and their probable associations with preterm birth judged by prior domain knowledge. ${ }^{29}$

To examine the contribution of risk factors to preterm birth incidence in the entire study population over the last decade, we measured the population attributable risk fraction using formula (1), where $A F p_{i}$ is the population attributable risk fraction for risk factor $i, P F_{j}$ is the proportion of the total population and $R R_{j}$ is the risk ratio for the exposure category $j(j=1,2, \ldots, m)$ of risk factor $i(i=1,2, \ldots, n) . R R_{i}$ was approximated by using $O R_{i}$ to avoid overlap from different risk factors. ${ }^{30} 31 \mathrm{AFp}$ was then calculated using formula (2) to measure the total population attributable risk fraction across all risk factors.

$$
A F p_{i}=\frac{\sum_{1}^{m} P F_{\mathrm{j}} *\left(R R_{j}-1\right)}{1+\sum_{1}^{\mathrm{m}} P F_{\mathrm{j}} *\left(R R_{j}-1\right)}(1)
$$

$$
A F p=1-\prod_{i}^{\mathrm{n}}\left(1-A F p_{i}\right)(2)
$$

We evaluated sociodemographic changes after implementation of the universal two-child policy and their contributions to the variations of preterm birth rate with approaches: (1) calculate $P F$ for each selected factor in two time periods, (2) identify the risk of preterm birth for these factors with ORs in a logistic regression among births after the policy, (3) estimate $A F p$ for each factor before and after the policy ( $A F p_{\text {before }}$ was calculated by $P F_{\text {before }}$ and $O R_{\text {after }}$ whereas $A F p_{\text {after }}$ was calculated by $P F_{\text {after }}$ and $O R_{\text {after }}$ ) and (4) calculate the total contribution to the changes in preterm birth rate between two periods by multiplying $A F p$ with the preterm birth rate after the policy and subtracting the result before the policy with formula. $^{32032}$

$$
\text { Increased Rate }=A F p_{\text {after }} * \text { Rate }_{\text {after }}-A F p_{\text {before }} * \text { Rate }_{\text {after }}
$$

All analyses were conducted using Python software (V.3.6.6; Python Software Foundation). Alpha levels of $0.001,0.01$ and 0.05 indicated statistical significance for a two-tailed test separately. ${ }^{33}$ Missing values of several variables were included in the descriptive analysis but were removed from the logistic regression analyses.

\section{RESULTS}

\section{Preterm birth rates in Baoan, Shenzhen}

A total of 480845 births in Baoan, Shenzhen were identified in the Shenzhen Birth Registry Database from 2009 to 2018. Furthermore, 478044 (99.4\%) live births were included in the final study population after excluding $2801(0.6 \%)$ ineligible birth records: $2561(0.5 \%)$ stillbirths or births with unknown results, $182(0.04 \%)$ live births with maternal age under 13 years or over 50 years and $58(0.01 \%)$ live births with missing gestational age or gestational age lower than 22 weeks or higher than 46 weeks. There were $27829(5.8 \%)$ preterm births in Baoan from 2009 to 2018 with $312(0.07 \%)$ extremely preterm births, $2686(0.6 \%)$ very preterm births and $24831(5.2 \%)$ late preterm births, respectively. The rates of PROM-PTB, S-PTB and MI-PTB were $0.08 \%, 3.1 \%$ and $2.6 \%$, accounting for $1.42 \%, 53.8 \%$ and $44.8 \%$ of the overall preterm births, respectively. The overall preterm birth rates and subtype-specific preterm birth rates among the different exposure categories for each maternal and infant group are presented in table 1.

\section{Temporal trends in preterm birth rate}

The annual overall preterm birth rates for the uncategorised study population and each exposure category of the selected risk factors during 2009-2018 are shown in figure 1 (see online supplemental table 1). The overall preterm birth rate fluctuated between $5.5 \%$ and $5.8 \%$ during 2009-2015 and surpassed $6.0 \%$ in 2016, reaching the highest rate in 2017 (6.4\%, figure 1A). Spontaneous preterm birth rates decreased, with an approximately 
Table 1 Descriptive statistics of preterm birth subtypes in Baoan, Shenzhen, 2009-2018

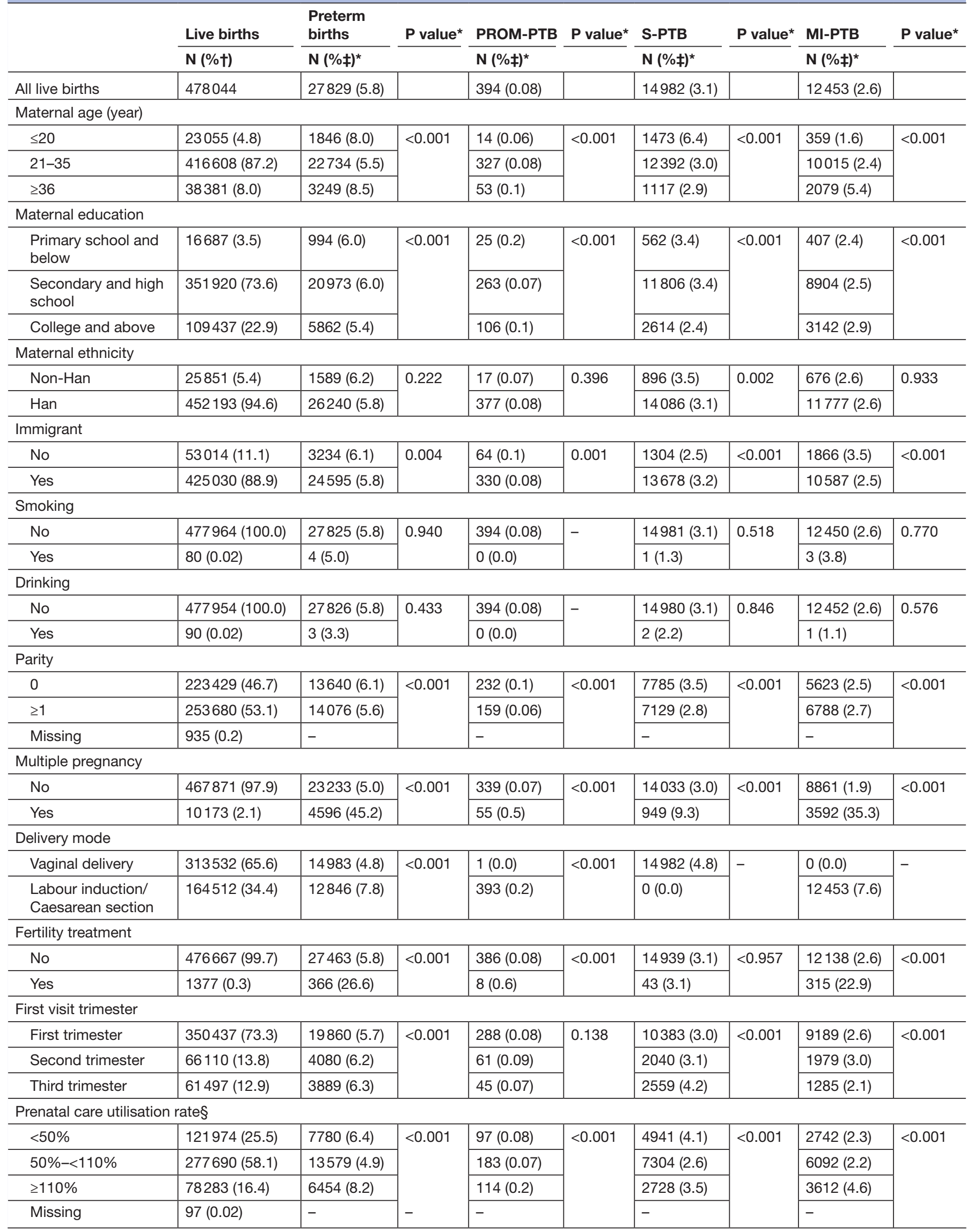


Table 1 Continued

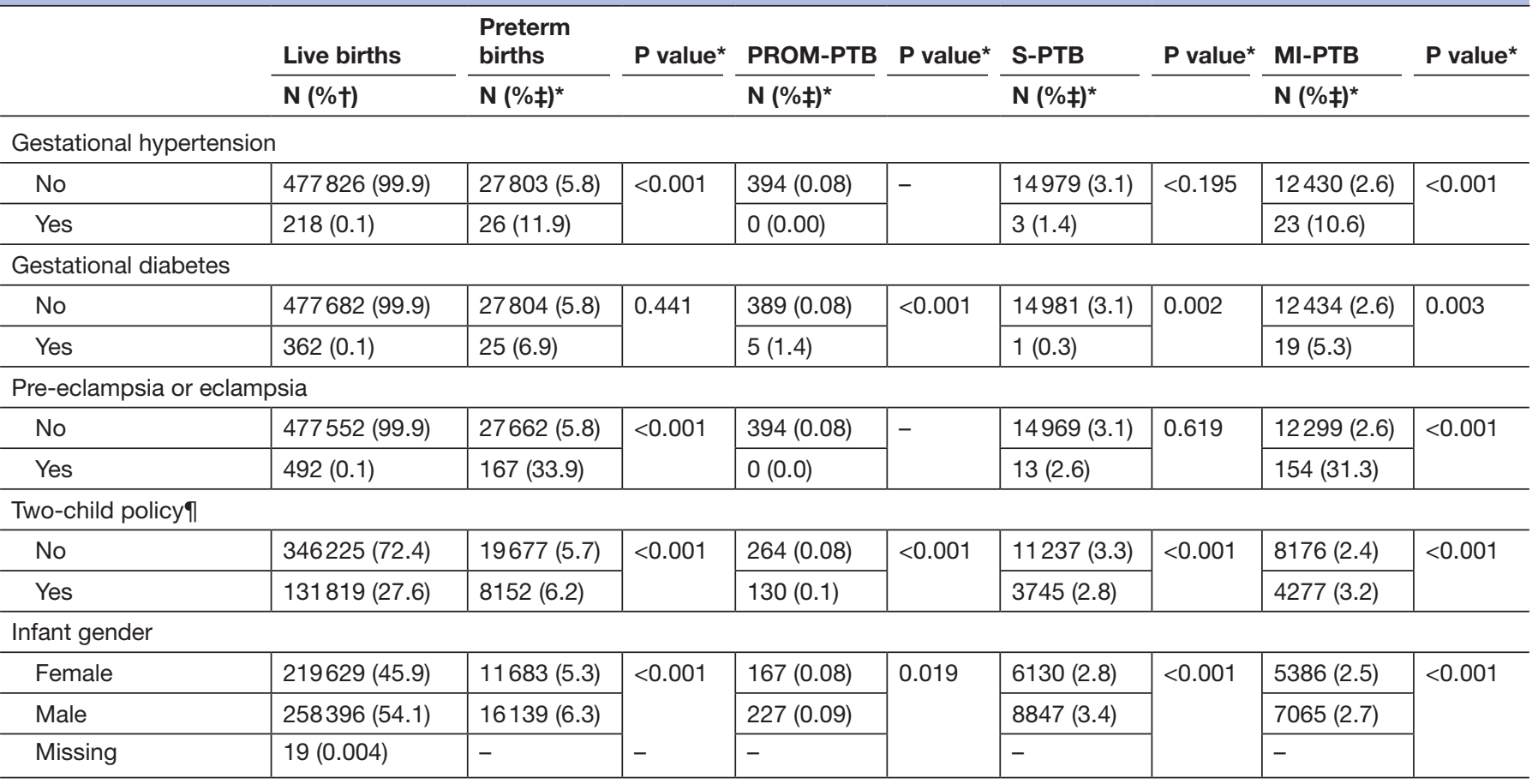

*Preterm birth frequencies among subcategories of each variable were compared with the $\mathrm{X}^{2}$ test.

†Distributions of maternal characteristics among the whole study population were calculated by the number of women in each subcategory divided by the total number of women, 478044 .

fOverall and subtype preterm birth rates were calculated by the number of preterm births divided by the number of women in each subcategory.

§Prenatal care utilisation rate is defined as the ratio between the actual number of visits and the recommended number.

IThe universal two-child policy effect time is defined as the delivery time before 1 July 2016, 9 months after the policy was announced in October 2015.

MI-PTB, medically induced preterm birth; PROM-PTB, premature rupture of membranes preterm birth ; S-PTB, spontaneous preterm birth.
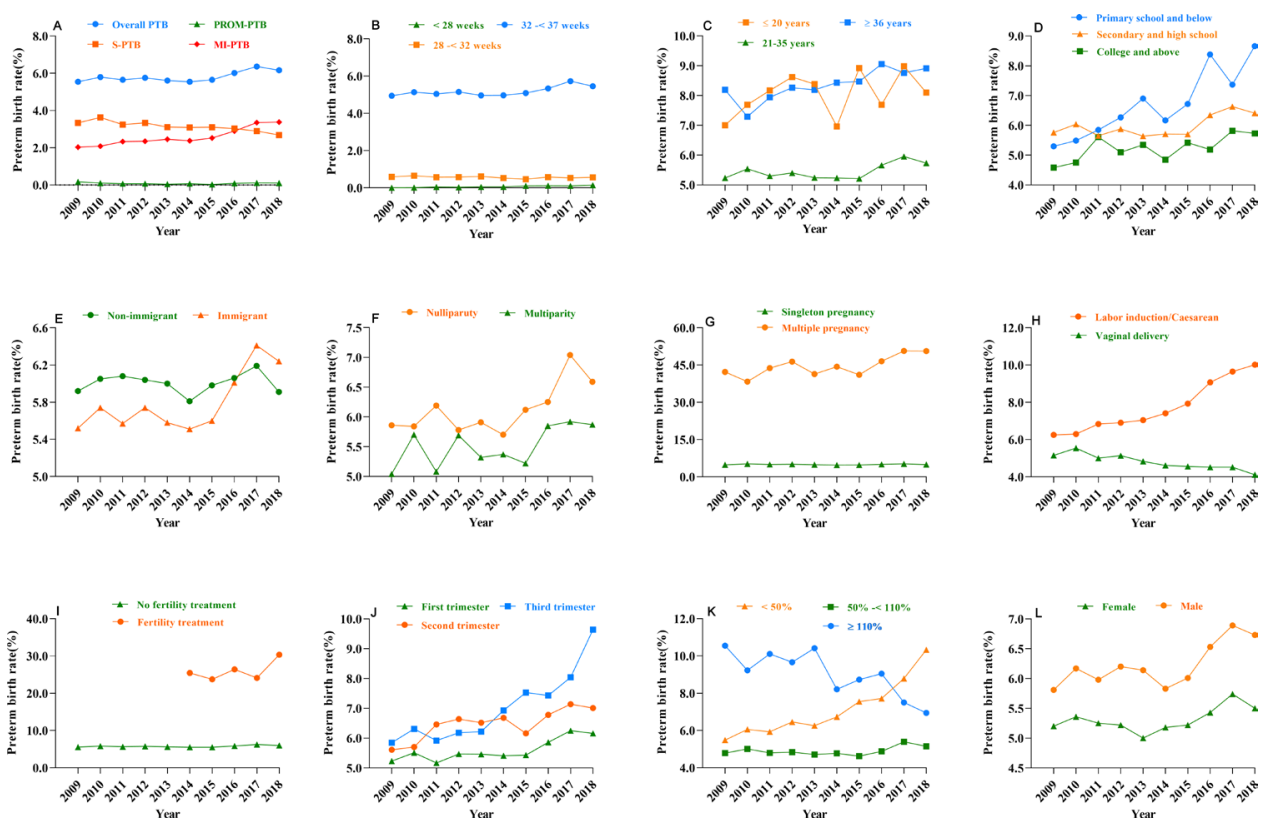

Figure 1 Temporal trends in preterm birth rate among 478044 livebirths (2801 ineligible birth records were excluded). Subcategorised by risk factors in Baoan, Shenzhen, 2009-2018. (A) overall and subtypes, (B) gestational age, (C) maternal age, (D) maternal education, (E) immigration, (F) parity, (G) multiple pregnancy, (H) delivery mode, (I) fertility treatment, (J) first visit trimester, $(\mathrm{K})$ prenatal care utilisation and $(\mathrm{L})$ infant gender. 
$0.07 \%$ decline in PROM-PTB and a $0.7 \%$ decline in S-PTB. However, the MI-PTB rate had increased year by year, from $2.0 \%$ in 2009 to $3.4 \%$ in 2018 (figure 1A). Annual changes in late preterm were more obvious than those in extremely preterm and very preterm infants (figure 1B). Generally, the rising trends in preterm birth were observed in exposure subcategories of maternal age (figure 1C), maternal education (figure 1D), immigration (figure 1E), parity (figure $1 \mathrm{~F}$ ), multiple pregnancy (figure 1G), labour induction or caesarean delivery mode (figure 1H), fertility treatment (figure 1I), first visit trimester (figure 1J), inadequate prenatal care utilisation (figure 1K) and infant $\operatorname{sex}$ (figure 1L). The results of the sensitivity analysis (see online supplemental table 1) indicated that statistically significant changes in annual preterm birth rates occurred only in the years 2016, 2017 and 2018 compared with 2009. The overall preterm birth trends for smoking, drinking, gestational hypertension, gestational diabetes, pre-eclampsia or eclampsia were not measured as they made up a small proportion in the positive results.

\section{Risk factors for preterm birth}

Based on the results of the $\chi^{2}$ test of possible maternal characteristics in table 1, statistically significant risk factors for both overall preterm birth and subtypes of preterm birth including maternal age, education level, immigration, parity, multiple pregnancy, fertility treatment, first prenatal care visit trimester, prenatal care utilisation, two-child policy and infant sex were further analysed using multivariable logistic regression. Gestational hypertension, diabetes and pre-eclampsia or eclampsia were not included in the regression model as they made up a small proportion in subgroups of preterm birth, even with significant associations with preterm birth (table 1). The corresponding adjusted ORs for each characteristic are presented in table 2. Maternal age, maternal education, parity, multiple pregnancy, prenatal care utilisation and infant gender still showed significant associations with both overall preterm birth and subtypes of preterm birth. For example, women with multiple pregnancy had a higher risk of preterm than the reference group (adjusted OR (AOR): 15.2, 95\% CI: 14.6 to 15.9; $\mathrm{p}<0.001$ ) with the highest risk of MI-PTB (adjusted OR (AOR): 25.6, 95\% CI: 24.4 to 26.8; $\mathrm{p}<0.001$ ) and lower risk of PROM-PTB (AOR: 5.7, 95\% CI: 4.2 to $7.7 ; \mathrm{p}<0.001$ ) and S-PTB (AOR: 3.5, 95\% CI: 3.3 to 3.8; $\mathrm{p}<0.001$ ). Births with maternal fertility treatment had a much higher risk of preterm birth rate, especially PROM-PTB rate and MI-PTB rate (table 1), but the strength of association was reduced by other risk factors in our multivariable logistic regression analysis (table 2).

\section{Temporal trends in the distribution of sociodemographic factors}

The distributions of sociodemographic factors including maternal age, education, immigration, parity, multiple pregnancy, delivery mode, first prenatal care visit trimester, prenatal care utilisation and infant sex are shown in figure 2 (see online supplemental table 3). Preterm births and the increase in preterm birth rate mainly occurred in the late preterm group (gestational age 32-<37weeks, figure 2A). The advanced maternal age group ( $\geq 36$ years) expanded from $6.2 \%$ in 2009 to $11.9 \%$ in 2018 (figure 2B). Improvement in maternal educational attainment is presented in figure $2 \mathrm{C}$, showing that the proportion of women with education level of primary school and below decreased from $17.6 \%$ in 2009 to $1.2 \%$ in 2018 . The percentage of immigrants decreased yearly during the decade from $95.8 \%$ in 2009 to $77.2 \%$ in 2018 (figure 2D). However, multiparity group expanded during the study period, from $39.5 \%$ in 2009 to $59.5 \%$ in 2018 (figure 2E). Women with labour induction or caesarean section accounted for approximately $35 \%$ of the entire population over the decade (figure $2 \mathrm{~F}$ ). The proportion of women who initiated prenatal care visits in the first trimester of pregnancy increased from 39.9\% in 2009 to $91.7 \%$ in 2018 (figure 2G). Prenatal care utilisation improved with a drastic increase in the proportion of adequate plus utilisation group from $5.4 \%$ in 2009 to $44.8 \%$ in 2018 (figure $2 \mathrm{H}$ ). The male-to-female sex ratio in Baoan, Shenzhen stayed around 117:100 over the decade in our study (figure 2I). Since 2014, fertility treatment had become slightly more prevalent among live births in Baoan, from no cases during 2009-2013 to $0.1 \%$ in 2014 and $0.7 \%$ in 2018 (see online supplemental table 3).

\section{Contributions of transitions in sociodemographic factors to variations in preterm birth rate}

The preterm birth rate of $5.7 \%$ during 1 January 2009 and 30 June 2016 increased to $6.2 \%$ during 1 July 2016 and 31 December 2018 , with $88 \%$ of the increase attributed to late preterm birth. The increase in the overall preterm rate mainly came from the increase in MI-PTB, while rate of preterm labour (S-PTB) decreased after June 2016. We compared preterm birth rates subcategorised by sociodemographic factors including maternal age, maternal education, parity, multiple pregnancy, prenatal care utilisation and infant sex, as well as the per cent compositions of these factors (table 3). Except for the adequate plus group of prenatal care utilisation, preterm birth rates increased in all the categories after the policy. The contributions of sociodemographic factors to the variations in the overall and subtypes of preterm birth rates between the two periods are visualised with population attributable risk fraction in figure 3 (online supplemental table 4). Maternal age and multiple pregnancy were drivers underlying the increment of the overall preterm birth rate, whereas maternal education, parity, prenatal care utilisation and infant sex contributed to the rate reduction. In particular, maternal education level increased, especially in the group of college and above, from $18.0 \%$ to $35.8 \%$ and an attributed $0.22 \%$ reduction in the overall preterm birth rate, a $0.14 \%$ decrease in S-PTB and a $0.05 \%$ decline in MI-PTB rate were evaluated, respectively. Births with inadequate prenatal care utilisation $(<50 \%)$ decreased from $32.2 \%$ to $7.7 \%$, which contributed to a $0.45 \%$ decrease in the overall preterm 
Table 2 Multivariable logistic regression of risk factors for overall preterm birth and subtypes of preterm birth in Baoan, Shenzhen, 2009-2018*

\begin{tabular}{|c|c|c|c|c|c|c|c|c|}
\hline & \multicolumn{2}{|c|}{ Overall preterm birth } & \multicolumn{2}{|c|}{ PROM-PTB } & \multicolumn{2}{|c|}{ S-PTB } & \multicolumn{2}{|c|}{ MI-PTB } \\
\hline & $\beta \dagger$ & AOR $(95 \% \mathrm{Cl}) \neq$ § П & $\beta \dagger$ & AOR $(95 \% \mathrm{Cl}) \ddagger$ § П & $\beta \dagger$ & AOR $(95 \% \mathrm{Cl}) \neq$ § П & $\beta \dagger$ & AOR $(95 \% \mathrm{Cl}) \neq$ § П \\
\hline \multicolumn{9}{|l|}{ Maternal age (year) } \\
\hline $21-35$ & - & Reference & - & Reference & - & Reference & - & Reference \\
\hline$\geq 36$ & 0.42 & $1.5(1.5$ to 1.6$) \ddagger$ & 0.55 & $1.7(1.3$ to 2.4$) \ddagger$ & 0.01 & $1.0(1.0$ to 1.1$)$ & 0.72 & $2.1(1.9$ to 2.2$) \ddagger$ \\
\hline $\begin{array}{l}\text { Secondary and } \\
\text { high school }\end{array}$ & 0.22 & $1.2(1.2$ to 1.3$) \ddagger$ & 0.11 & 1.1 (0.9 to 1.5$)$ & 0.31 & 1.4 (1.3 to 1.4$) \ddagger$ & 0.08 & $1.1(1.0$ to 1.1$)$ q \\
\hline College and above & - & Reference & - & Reference & - & Reference & - & Reference \\
\hline \multicolumn{9}{|l|}{ Immigrant } \\
\hline 0 & - & Reference & - & Reference & - & Reference & - & Reference \\
\hline$\geq 1$ & -0.09 & 0.9 (0.9 to 0.9$) \ddagger$ & -0.56 & 0.6 (0.5 to 0.7$) \ddagger$ & -0.18 & 0.8 (0.8 to 0.9$) \ddagger$ & 0.07 & 1.1 (1.0 to 1.1$)$ १ \\
\hline \multicolumn{9}{|l|}{ Multiple pregnancy } \\
\hline No & - & Reference & - & Reference & - & Reference & - & Reference \\
\hline Yes & 2.72 & 15.2 (14.6 to 15.9$) \ddagger$ & 1.74 & 5.7 (4.2 to 7.7$) \ddagger$ & 1.25 & 3.5 (3.3 to 3.8$) \ddagger$ & 3.24 & 25.6 (24.4 to 26.8$) \neq$ \\
\hline \multicolumn{9}{|l|}{ Fertility treatment } \\
\hline No & - & Reference & - & Reference & - & Reference & - & Reference \\
\hline Yes & -0.01 & 1.0 (0.9 to 1.1$)$ & 0.39 & 1.5 (0.7 to 3.1$)$ & -0.75 & 0.5 (0.4 to 0.7$) \ddagger$ & 0.16 & 1.2 (1.0 to 1.4$) \S$ \\
\hline \multicolumn{9}{|l|}{ First visit trimester } \\
\hline$\geq 110 \%$ & - & Reference & - & Reference & - & Reference & - & Reference \\
\hline \multicolumn{9}{|l|}{ Two-child policy†† } \\
\hline No & - & Reference & - & Reference & - & Reference & - & Reference \\
\hline Yes & 0.07 & 1.1 (1.0 to 1.1$) \ddagger$ & 0.19 & 1.2 (1.0 to 1.5$)$ & -0.03 & 1.0 (0.9 to 1.0$)$ & 0.16 & $1.2(1.1$ to 1.2$) \ddagger$ \\
\hline \multicolumn{9}{|l|}{ Infant gender } \\
\hline Female & - & Reference & - & Reference & - & Reference & - & Reference \\
\hline Male & 0.20 & $1.2(1.2$ to 1.3$) \ddagger$ & 0.17 & 1.2 (1.0 to 1.4$)$ & 0.22 & 1.3 (1.2 to 1.3$) \ddagger$ & 0.14 & $1.2(1.1$ to 1.2$) \ddagger$ \\
\hline
\end{tabular}

*476 997 live births were included after removing 1047 records due to missing values in any risk factor.

$\dagger \beta$, coefficients of risk factors in the multivariable binomial logistic regression model.

$\neq \mathrm{p}<0.001$.

$\S \mathrm{p}<0.05$.

१p $<0.01$.

${ }^{*}$ Prenatal care utilisation rate is defined as the ratio between the actual number of visits and the recommended number.

††The universal two-child policy is defined as the delivery time before 1 July 2016, 9 months after the policy was announced in October 2015. AOR, adjusted OR; MI-PTB, medically induced preterm birth; PROM-PTB, premature rupture of membranes preterm birth; S-PTB, spontaneous preterm birth.

birth rate, $0.01 \%$ increase of PROM-PTB rate, $0.27 \%$ decrease of S-PTB and $0.14 \%$ reduction of MI-PTB. Even with a small change in composition, from $2.0 \%$ to $2.5 \%$, multiple pregnancy had contributed to over half of the increase $(0.28 \% / 0.52 \%)$ in the overall preterm birth rate, with a major effect on MI-PTB. Maternal age contributed 

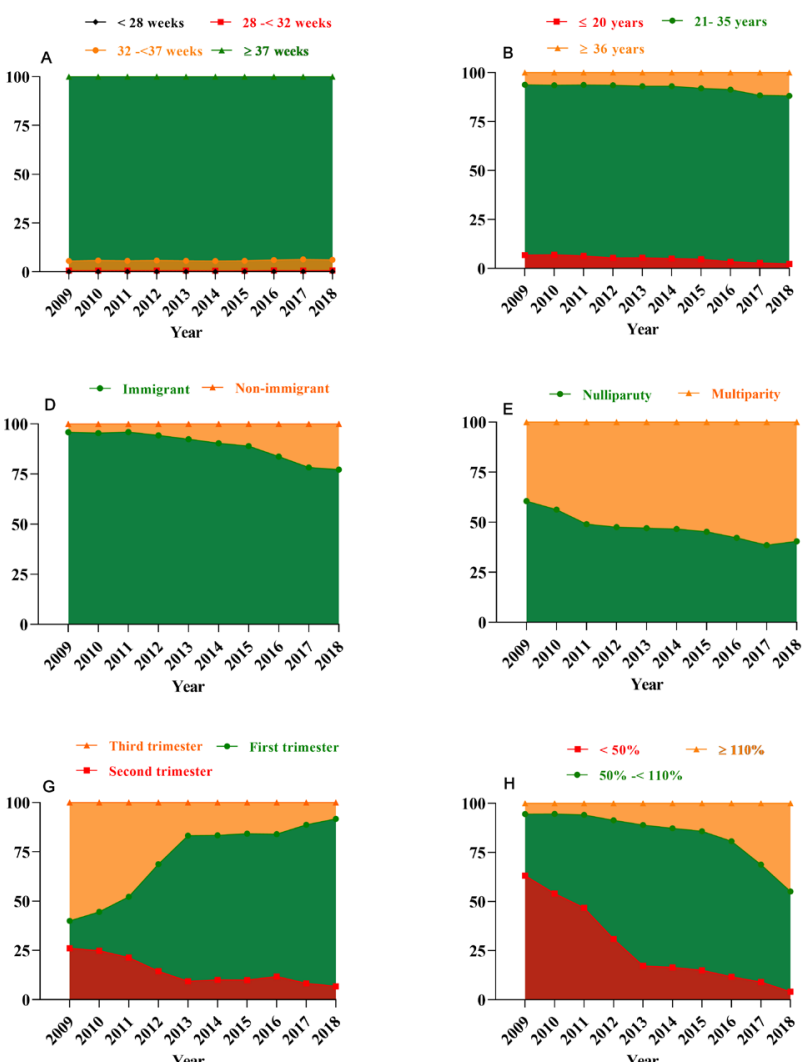
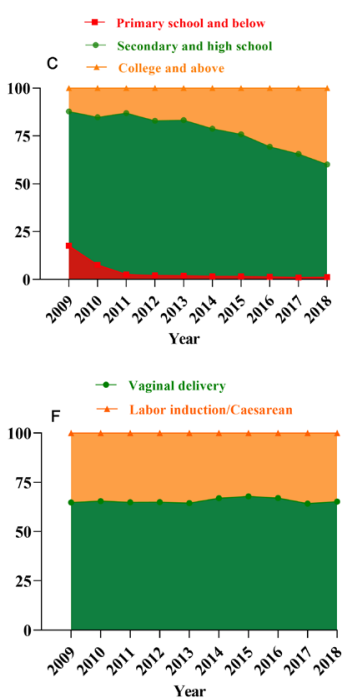

Year

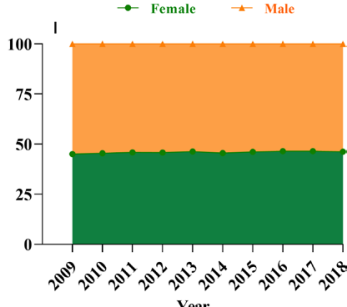

Figure 2 Temporal trends in the distribution of sociodemographic factors in Baoan, Shenzhen, 2009-2018. (A) gestational age, (B) maternal age, (C) maternal education, (D) immigration, (E) parity, $(F)$ delivery mode, $(G)$ first visit trimester, (H) prenatal care utilisation and (I) infant gender.

to an increase in the rate of MI-PTB but a decrease in the rate of S-PTB. The proportion of younger maternal age dropped from $5.6 \%$ to $2.8 \%$ while advanced maternal age ( $\geq 36$ years) grew from $6.9 \%$ to $11.1 \%$, contributing to a $0.03 \%$ increase in preterm birth rate. Multiparous births increased from $50.4 \%$ to $60.3 \%$ and had made a $0.06 \%$ decrease in preterm birth rate. The infant sex ratio remained stable during the two periods and its contribution to the change in preterm birth rate was very small.

\section{DISCUSSION}

To date, the persistent increase in preterm birth rate remains a challenging public health issue facing the world and limited studies have focused on the temporal trends of preterm birth during a sociodemographic transition period in recent decades. In this retrospective cohort study, a statistical analysis of 478044 birth records demonstrated the drivers of the time trends in preterm birth among a mixed population, under the background of sociodemographic transition in Shenzhen. The findings of this study highlight the importance of safeguarding the health and well-being of women to reduce preterm birth, especially through improving maternal education and prenatal care service coverage.

During the sociodemographic transition period from 2009 to 2018, the overall preterm birth rate of $5.8 \%$ in Baoan was at a relatively lower level compared with the global preterm birth rate ranging from 5\% in northern European countries to $18 \%$ in African countries. ${ }^{6}$ It is also lower than the weighted national incidence of $6.7 \%$ in China during 2015-2016. ${ }^{34}$ However, it was slightly higher than the Shenzhen preterm birth rate of 5.7\% during 2003-2012, and it was slightly higher during 2009-2018. ${ }^{20}$ Consistent with the global trend of the rising preterm birth rates reported by many countries in recent years, the prevalence of preterm birth in Baoan increased from 5.5\% in 2009 to $6.2 \%$ in 2018 under the background of sociodemographic transition. ${ }^{52}$ Compared with the whole Shenzhen birth population during 2003-2012, both the S-PTB rate and MI-PTB rate in Baoan increased during 2009-2018, while there was a $0.5 \%$ decrease in the PROM-PTB rate (see online supplemental table 5). ${ }^{20}$ MI-PTB and late preterm drove the majority of the increase in preterm birth rates both in our research and the study of the entire Shenzhen birth population during 2003-2012. ${ }^{20}$ In our analysis of risk factors, multiple pregnancy had a strong effect on preterm birth, especially MI-PTB and more than half of the increase in the overall preterm birth rate between the two periods (before and after implementation of the universal two-child policy) could be explained by it. Findings from other studies indicated that multiple pregnancy was a strong risk factor for preterm birth with a 7 to 10 times higher risk than that of singletons. ${ }^{35} 36$ Concerns have been raised in many studies about the increasing trend of multiple pregnancy reported both in China and worldwide, which was associated with the global rise in advanced maternal age, infertility treatments and obstetric interventions performed before 37 gestational weeks, especially at $34-36$ weeks. ${ }^{37-41}$ 
Table 3 Preterm birth rate and distribution of risk factors in Baoan, Shenzhen, 2009-2018*

\begin{tabular}{|c|c|c|c|c|}
\hline & \multicolumn{2}{|l|}{ Preterm birth rate (\%) } & \multicolumn{2}{|c|}{ Distribution percentage (\%)† } \\
\hline & $\begin{array}{l}\text { January } 2009 \text { to June } \\
2016\end{array}$ & $\begin{array}{l}\text { July } 2016 \text { to } \\
\text { December } 2018\end{array}$ & $\begin{array}{l}\text { January } 2009 \text { to June } \\
2016\end{array}$ & $\begin{array}{l}\text { July } 2016 \text { to } \\
\text { December } 2018\end{array}$ \\
\hline PROM-PTB & 0.08 & 0.1 & - & - \\
\hline S-PTB & 3.2 & 2.8 & - & - \\
\hline MI-PTB & 2.4 & 3.2 & - & - \\
\hline$<28$ & 0.05 & 0.1 & 0.8 & 1.8 \\
\hline $28-<32$ & 0.6 & 0.6 & 9.9 & 9.1 \\
\hline $32-<37$ & 5.1 & 5.5 & 89.3 & 89.2 \\
\hline \multicolumn{5}{|l|}{ Maternal age(year) } \\
\hline \multicolumn{5}{|l|}{ Maternal education } \\
\hline Primary school and below & 5.7 & 7.8 & 4.4 & 1.2 \\
\hline Secondary and high school & 5.8 & 6.5 & 77.6 & 63.1 \\
\hline College and above & 5.2 & 5.6 & 18.0 & 35.8 \\
\hline \multicolumn{5}{|l|}{ Parity } \\
\hline 0 & 5.9 & 6.6 & 49.6 & 39.7 \\
\hline$\geq 1$ & 5.4 & 5.9 & 50.4 & 60.3 \\
\hline \multicolumn{5}{|l|}{ Multiple pregnancy } \\
\hline No & 4.9 & 5.1 & 98.0 & 97.5 \\
\hline Female & 5.2 & 5.6 & 45.8 & 46.5 \\
\hline Male & 6.0 & 6.7 & 54.3 & 53.6 \\
\hline
\end{tabular}

*476997 live births were included after removing 1047 records due to missing values in any risk factor.

†The distribution percentage for each category is the number of cases divided by the total number of preterm births.

$\ddagger$ Prenatal care utilisation rate is defined as the ratio between the actual number of visits and the recommended number.

MI-PTB, medically induced preterm birth; PROM-PTB, premature rupture of membranes preterm birth; S-PTB, spontaneous preterm birth.

In contrast with multiple pregnancy, the improvements in maternal education and prenatal care utilisation have contributed to the reduction in the overall preterm birth rate as well as the subtype-specific preterm birth rates, which has coincided with socioeconomic developments in China with the launch of laws and policies, including 9-year compulsory education for all and national commitment to maternal and child survival and health. ${ }^{42}$ In particular, the proportion of pregnant women with inadequate prenatal care utilisation decreased after implementation of the universal two-child policy and contributed to a $0.5 \%$ decline in the preterm birth rate. The positive effect of prenatal care on preterm birth during 2009-2018 was estimated to be larger than the period 2003-2012 in the whole Shenzhen birth population. ${ }^{20}$ Although the proportion of women who initiated prenatal care in the first trimester increased from $39.9 \%$ in 2009 to $91.7 \%$ in 2018, this was not significantly associated with the overall and subtypes of preterm birth rates. As suggested by the Born Too Soon Group, further studies are needed to clarify the association between the quality of prenatal care visits and preterm birth. ${ }^{44}$ Additionally, the $0.22 \%$ of reduction in preterm birth rate could be explained by the expansion of maternal educational attainment during 2009-2018. However, the effect of maternal education on preterm birth in this study is contrary to the result in the Shenzhen preterm birth research during 2003-2012, which demonstrated that the education improvement had contributed to $0.20 \%$ of the rise in preterm birth rate. ${ }^{20}$ It should be noted that the proportion of multiparous births increased continuously over the decade, including and a near 10\% increment after 


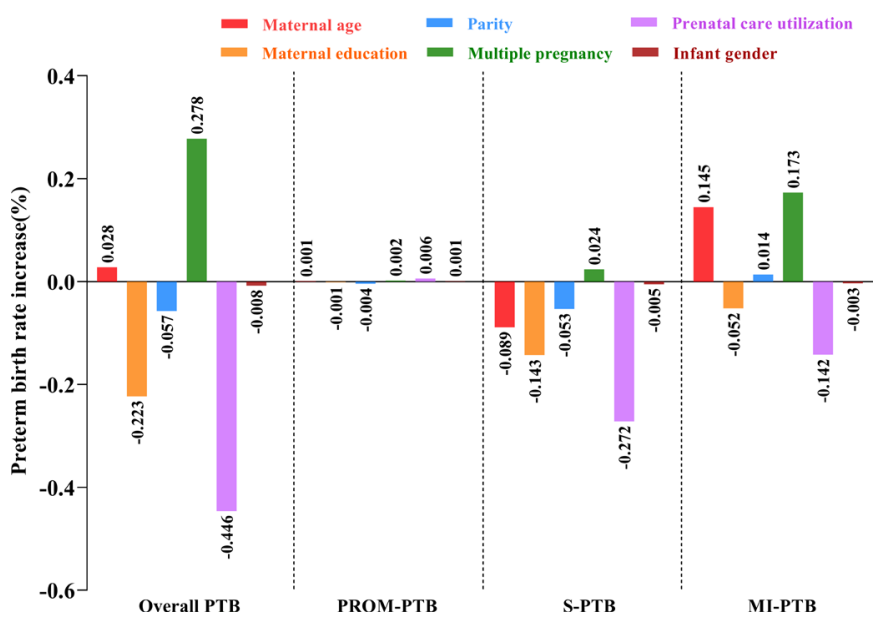

Figure 3 Analysis of sociodemographic factors contributing to the variations of preterm birth rate in Baoan, Shenzhen, 2009-2018. MI-PTB, medically induced preterm birth; PROMPTB, premature rupture of membranes preterm birth ; S-PTB, spontaneous preterm birth.

the implementation of the universal two-child policy, which resulted in a small reduction in the preterm birth rate. The percentage of multiparity in this study was approximately $15.3 \%$ higher than that in the Shenzhen birth population during 2003-2012 and also higher than the national level. $^{2025}$ The male-to-female sex ratio in Baoan during 2009-2018 remained abnormal compared with the natural sex ratio at birth, which indicated that a more balanced sex ratio, one of the expected benefits of the universal two-child policy, had not yet been achieved. ${ }^{454}$

The present study is unique in reporting time trends in preterm birth under a setting of rapid sociodemographic transition over 10years. Contributions of sociodemographic factors to preterm birth and to the incidence changes were calculated to provide a more comprehensive and quantitative understanding of the pathogenesis of temporal trends in preterm birth. Overall and subtypes of preterm birth, including spontaneous preterm and iatrogenic preterm, were analysed respectively. Per cent compositions of sociodemographic factors in each year have been presented to provide a better landscape of the socio-economic transition in this area. Missing information on prenatal care utilisation and parity were unlikely to have impacted the results.

However, the study results were limited by data collection and analysis methods. First, misclassification of preterm birth subtypes was possible because subtype of preterm birth was unavailable in the Shenzhen Birth Registry Database. Fortunately, the classification method based on delivery mode and surgical indications has been adapted by similar research and reliability of the database has been verified by previous study. ${ }^{19} 20$ Second, there were many important factors we did not cover in this study as the limitation of the database. For example, data on maternal employment, family income and maternal obesity were not available. Increased risks of preterm birth were significantly related to maternal obesity in a cohort study of 1599551 live singleton births in Sweden from 1992 to $2010 .{ }^{47}$ Similar results were also found in a nationwide study of 7141630 singleton live births from the USA during 2016 and $2017 .{ }^{48}$ Finally, sociodemographic transitions in maternal age and multiple pregnancy have been identified as contributors to the rising preterm birth rate in Baoan, Shenzhen during 2009-2018 in this study. More possible important factors, including maternal employment, family income, non-medically indicated labour, induction and caesarean section deliveries, assisted reproductive technologies need to be studied to present a more comprehensive understanding about the impact of sociodemographic transitions on preterm birth.

\section{CONCLUSIONS}

In conclusion, the present study provides unique information about the temporal trends in preterm birth in the setting of rapid sociodemographic transition in China during the last decade. The observed increase in preterm birth rate was significantly associated with the increase of multiple pregnancy. Fortunately, maternal educational attainment and prenatal care utilisation have improved significantly during this period, and have made positive contributions to the decline in preterm birth incidence. The study findings highlight that the investment in girls' education, quality reproductive and maternal healthcare may significantly reduce the rate of babies born too soon and the economic burden of preterm birth. More studies need to be conducted to discover the hidden risk factors that drive the increase in preterm birth rate and finally to reduce the prevalence of preterm birth and its global burden.

Acknowledgements We would like to thank the staff who provided us with limited but accredited access to the Shenzhen Birth Registry Database and suggestions on the use of this database.

Contributors LZ, LZh and RM developed the study concept and design. RM, YL and QX collected data. RM cleaned the data and performed data analysis. RM and LZ drafted the manuscript. LZ, LZh, YL, JW, YZ, HS and XR provided oversight and expert advice for the research and the written paper. All authors revised and approved the final paper.

Funding This study is supported by the National Natural Science Foundation of China (grant number: 31771468 ).

\section{Competing interests None declared.}

Patient and public involvement Patients and/or the public were not involved in the design, or conduct, or reporting or dissemination plans of this research.

\section{Patient consent for publication Not required.}

Ethics approval This study was approved by the medical ethics committee of Shenzhen Baoan Women's and Children's Hospital, Jinan University, China (number LLSC-2019-07-01). All data used in this study have been completely anonymised before accession and were analysed anonymously.

Provenance and peer review Not commissioned; externally peer reviewed.

Data availability statement Data are available upon reasonable request to the corresponding author.

Open access This is an open access article distributed in accordance with the Creative Commons Attribution Non Commercial (CC BY-NC 4.0) license, which permits others to distribute, remix, adapt, build upon this work non-commercially, and license their derivative works on different terms, provided the original work is properly cited, appropriate credit is given, any changes made indicated, and the use is non-commercial. See: http://creativecommons.org/licenses/by-nc/4.0/.

\section{ORCID iDs}


Rui Ma http://orcid.org/0000-0003-0910-3164

Lingyun Zou http://orcid.org/0000-0002-1109-5709

\section{REFERENCES}

1 Romero R, Dey SK, Fisher SJ. Preterm labor: one syndrome, many causes. Science 2014;345:760-5.

2 Maghsoudlou S, Yu ZM, Beyene J, et al. Phenotypic classification of preterm birth among nulliparous women: a population-based cohort study. J Obstet Gynaecol Can 2019;41:1423-32.

3 Lawn JE, Kinney MV, Belizan JM, et al. Born too soon: accelerating actions for prevention and care of 15 million newborns born too soon. Reprod Health 2013;10:S6.

4 United Nations Inter-agency Group for Child Mortality Estimation (UNIGME). Levels \& trends in child mortality: report 2019, estimates developed by the United Nations inter-agency group for child mortality estimation United Nations children's fund, New York, 2019.

5 Chawanpaiboon S, Vogel JP, Moller A-B, et al. Global, regional, and national estimates of levels of preterm birth in 2014: a systematic review and modelling analysis. Lancet Glob Health 2019;7:e37-46.

6 Blencowe H, Cousens S, Chou D, et al. Born too soon: the global epidemiology of 15 million preterm births. Reprod Health 2013;10:S2.

7 Behrman RE, Butler AS, Institute of Medicine (US) Committee on Understanding Premature Birth and Assuring Healthy Outcomes. Preterm birth: causes, consequences, and prevention. National Academies Press, 2007.

8 Johnston KM, Gooch K, Korol E, et al. The economic burden of prematurity in Canada. BMC Pediatr 2014;14:93.

9 Countdown to 2030 Collaboration, Boerma T. Countdown to 2030: tracking progress towards universal coverage for reproductive, maternal, newborn, and child health. Lancet 2018;391:1538-48.

10 Rahman A, Rahman M, Pervin J, et al. Time trends and sociodemographic determinants of preterm births in pregnancy cohorts in Matlab, Bangladesh, 1990-2014. BMJ Glob Health 2019;4:e001462.

11 Sakata S, Konishi S, Ng CFS, et al. Preterm birth rates in Japan from 1979 to 2014: analysis of national vital statistics. J Obstet Gynaecol Res 2018;44:390-6.

12 López Orellana P. Increase in preterm birth during demographic transition in Chile from 1991 to 2012. Biomed Res Int 2015;2015:845968

13 Sadovsky ADIde, Matijasevich A, Santos IS, et al. Socioeconomic inequality in preterm birth in four Brazilian birth cohort studies. $J$ Pediatr 2018;94:15-22.

14 Glinianaia SV, Ghosh R, Rankin J, et al. No improvement in socioeconomic inequalities in birthweight and preterm birth over four decades: a population-based cohort study. BMC Public Health 2013;13:345

15 Yang M, Dijst M, Helbich M. Mental health among migrants in Shenzhen, China: does it matter whether the migrant population is identified by Hukou or birthplace? Int J Environ Res Public Health 2018;15:2671.

16 Shenzhen Statistics Bureau. Shenzhen statistical yearbook 2019. Available: http://www.sz.gov.cn/attachment/0/405/405056/6616865. pdf [Accessed 30 Dec 2019].

17 Shenzhen Baoan Statistics Bureau. Shenzhen Baoan statistical yearbook 2017. Available: http://sso.sz.gov.cn/pub/baqzfzx2017/tjj/ ywgz_136262/tjsj_137617/tjnj_137621/201811/P020181130433800 362927.pdf [Accessed 30 Nov 2018].

18 Shenzhen Baoan Statistics Bureau. Shenzhen Baoan statistical yearbook 2012. Available: http://sso.sz.gov.cn/pub/baqzfzx2017/tjj/ ywgz_136262/tjsj_137617/tjnj_137621/201404/P020140429367563 038549.pdf [Accessed 24 Feb 2014].

19 Liang Z, Lin Y, Ma Y, et al. The association between ambient temperature and preterm birth in Shenzhen, China: a distributed lag non-linear time series analysis. Environ Health 2016;15:84.

20 Li C, Liang Z, Bloom MS, et al. Temporal trends of preterm birth in Shenzhen, China: a retrospective study. Reprod Health 2018;15:47.

21 WHO. WHO: recommended definitions, terminology and format for statistical tables related to the perinatal period and use of a new certificate for cause of perinatal deaths. modifications recommended by FIGO as amended October 14, 1976. Acta Obstet Gynecol Scand 1977;56:247-53

22 Tang $\mathrm{K}$, Wang $\mathrm{H}$, Tan $\mathrm{SH}$, et al. Association between maternal education and breast feeding practices in China: a population-based cross-sectional study. BMJ Open 2019;9:e028485.
23 Zhao Q, Huang ZJ, Yang S, et al. The utilization of antenatal care among rural-to-urban migrant women in Shanghai: a hospital-based cross-sectional study. BMC Public Health 2012;12:1012.

24 Osterman MJK, Martin JA. Timing and adequacy of prenatal care in the United States, 2016. Natl Vital Stat Rep 2018;67:1-14.

25 Li H-T, Xue M, Hellerstein S, et al. Association of China's universal two child policy with changes in births and birth related health factors: national, descriptive comparative study. BMJ 2019;366:14680.

$26 \mathrm{McHugh}$ ML. The chi-square test of independence. Biochem Med 2013;23:143-9.

27 Thabane L, Mbuagbaw L, Zhang S, et al. A tutorial on sensitivity analyses in clinical trials: the what, why, when and how. BMC Med Res Methodol 2013;13:92.

28 Sperandei S. Understanding logistic regression analysis. Biochem Med 2014;24:12-18.

29 Goldenberg RL, Culhane JF, lams JD, et al. Epidemiology and causes of preterm birth. Lancet 2008;371:75-84.

30 Auger N, Le TUN, Park AL, et al. Association between maternal comorbidity and preterm birth by severity and clinical subtype: retrospective cohort study. BMC Pregnancy Childbirth 2011;11:67.

31 VanderWeele TJ, Lantos JD, Lauderdale DS. Rising preterm birth rates, 1989-2004: changing demographics or changing obstetric practice? Soc Sci Med 2012;74:196-201.

32 Chang $\mathrm{HH}$, Larson J, Blencowe $\mathrm{H}$, et al. Preventing preterm births: analysis of trends and potential reductions with interventions in 39 countries with very high human development index. Lancet 2013;381:223-34.

33 Greenland S, Senn SJ, Rothman KJ, et al. Statistical tests, P values, confidence intervals, and power: a guide to misinterpretations. Eur $J$ Epidemiol 2016;31:337-50.

34 Chen C, Zhang J, Xia H, et al. Epidemiology of preterm birth in China in 2015 and 2016: a nationwide survey. Lancet 2018;392:S73.

35 Dodd JM, Grivell RM, OBrien CM, et al. Prenatal administration of progestogens for preventing spontaneous preterm birth in women with a multiple pregnancy. Cochrane Database Syst Rev 2019;2019

36 Blondel B, Macfarlane A, Gissler M, et al. Preterm birth and multiple pregnancy in European countries participating in the PERISTAT project. BJOG 2006;113:528-35.

37 Deng C, Dai L, Yi L, et al. Temporal trends in the birth rates and perinatal mortality of twins: a population-based study in China. PLoS One 2019;14:e0209962.

38 Pison G, Monden C, Smits J. Twinning rates in developed countries: trends and explanations. Popul Dev Rev 2015;41:629-49.

39 Richards JL, Kramer MS, Deb-Rinker P, et al. Temporal trends in late preterm and early term birth rates in 6 high-income countries in North America and Europe and association with clinician-initiated obstetric interventions. JAMA 2016;316:410-9.

40 Fox $\mathrm{H}$, Callander E, Lindsay D, et al. Evidence of overuse? patterns of obstetric interventions during labour and birth among Australian mothers. BMC Pregnancy Childbirth 2019;19:226.

41 Zhong N. Achieving lower preterm birth rates in China via reductions in iatrogenic preterm births. Am J Public Health 2019;109:1489-90.

42 He C, Liu L, Chu Y, et al. National and subnational all-cause and cause-specific child mortality in China, 1996-2015: a systematic analysis with implications for the sustainable development goals. Lancet Glob Health 2017;5:e186-97.

43 Guo Y, Bai J, Na H. The history of China's maternal and child health care development. Semin Fetal Neonatal Med 2015;20:309-14.

44 Requejo J, Merialdi M, Althabe F, et al. Born too soon: care during pregnancy and childbirth to reduce preterm deliveries and improve health outcomes of the preterm baby. Reprod Health 2013;10:S4.

45 Shah PS. Knowledge synthesis group on determinants of LBWPTb. parity and low birth weight and preterm birth: a systematic review and meta-analyses. Acta Obstet Gynecol Scand 2010;89:862-75.

46 Zeng Y, Hesketh T. The effects of China's universal two-child policy. Lancet 2016;388:1930-8.

47 Cnattingius S, Villamor E, Johansson S, et al. Maternal obesity and risk of preterm delivery. JAMA 2013;309:2362-70.

48 Liu B, Xu G, Sun Y, et al. Association between maternal prepregnancy obesity and preterm birth according to maternal age and race or ethnicity: a population-based study. Lancet Diabetes Endocrinol 2019;7:707-14 